\title{
EARTHING SYSTEM EVALUATION AND INFLUENCE ON PROTECTION PERFORMANCE IN RESONANTLY EARTHED MV NETWORKS
}

\author{
Sandra HUTTER \\ HEP ODS d.o.o., Elektra Zagreb - Croatia \\ sandra.hutter@hep.hr
}

\begin{abstract}
In Zagreb distribution area, Petersen coils are installed in five 110/10(20) $\mathrm{kV}$ substations. The advantages of resonantly earthed $M V$ networks (continuous operation, arc self-extinction etc.) and some disadvantages (lower protection sensitivity) are well known. An overview of the procedure implemented before putting Peterson coils in operation is given, with emphasis on the earthing system evaluation, which has proved to be rather important. Protection testing should be performed for different types of faults. All data are given for two distribution areas in which Peterson coils have been recently installed.
\end{abstract}

\section{INTRODUCTION}

High capacitive short circuit currents of 186 A and 114 A were measured in two 123/10(20) kV substations (Fig. 1). In previous years, continued recording of capacitive short circuit currents in those substations have shown an allover increasing trend, with an increase of 7-10 A per year. As a possible solution resonant earthing with Petersen coils of $3603 \mathrm{kVA}$ and $5000 \mathrm{kVA}$ was proposed which should insure a needed compensation level in following years as well.

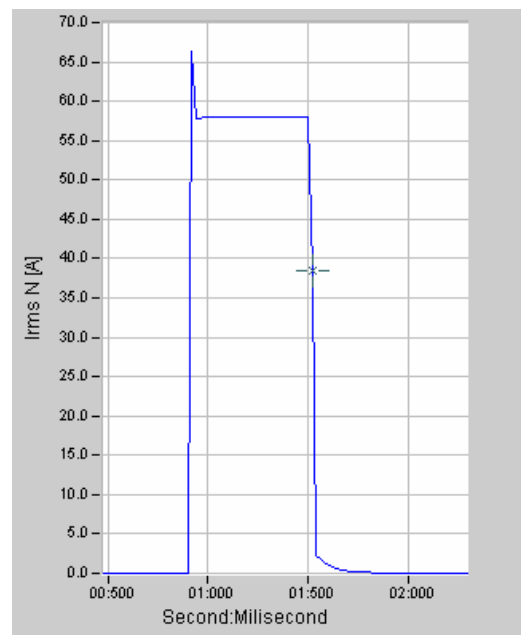

Fig. 1. Measured rms values of capacitive short circuit currents in the substation A (transformer I with busbar system I)

\section{NEUTRAL POINT EARTHING}

The plan used to implement neutral point earthing will directly influence the quality of the network operation, as well as on the type (and price) of the installed equipment and on the choice of protection. If the network is earthed over a small resistance, the resistive component of the short circuit current will have to be greater than the total capacitive component of the short circuit current.

\section{Resonantly earthed MV network}

If the MV network is operated with a resonantly earthed resonant point, supply interruption is greatly reduced, resulting in improved power quality, continuous operation even during the short circuit conditions in the network and a lower potential rise of the earthing systems [1]. Petersen coils' inductance can be continually tuned up and thus give better results regarding the total number of interruptions, however their maintenance during network operation can be more challenging. The level of compensation has to be periodically checked in order to improve their ability to „override“ the faults in the network (Fig. 2.).

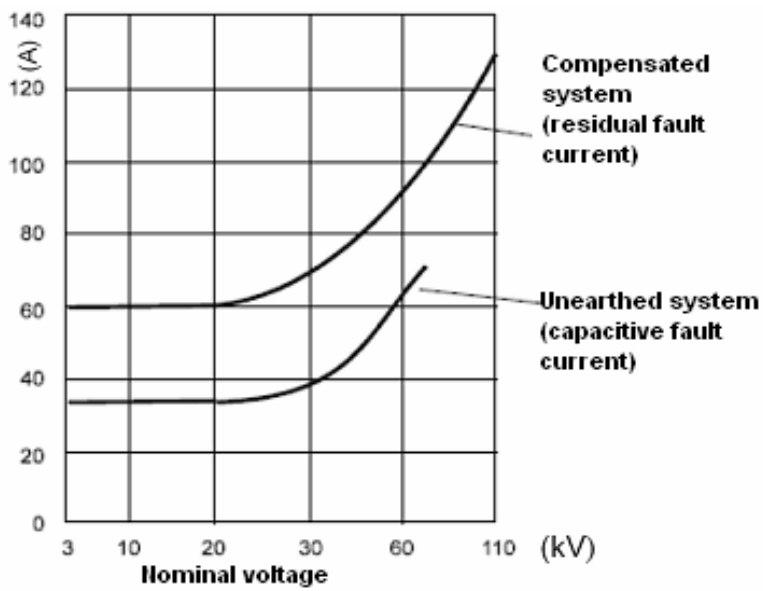

Fig. 2. Current limits of earth fault extinction according to DIN VDE 0228, Part 2 [2]

\section{DISTRIBUTION AREAS}

Both distribution networks supply smaller towns in Zagreb's proximity as well as part of the rural networks with long over head lines. Due to their proximity to Zagreb both areas have the potential to develop small to medium industrial utilities, thus increasing the need for the 
expansion of the cable network. The feeders are principally radial. One part of the distribution area $\mathrm{A}$ is located further away in a hard to reach, hilly countryside with poor soil quality, while distribution area B consists of a wide plane area with good soil quality.

\section{Statistics for the Substation A}

The total length of the network is $190 \mathrm{~km}, 78 \mathrm{~km}$ out of which are covered by over head lines with 68 secondary distribution substations $10 / 0.4 \mathrm{kV}$. The length of the cable network is $112 \mathrm{~km}$ with 125 secondary distribution substations $10 / 0.4 \mathrm{kV}$.

A total number of 55 interruptions lasting longer than 3 minutes were recorded in 2005 (over current protection has operated 6 times and short circuit protection has operated 49 times). The total number of interruptions has decreased to 33 in 2006 (over current protection has operated 5 times and short circuit protection has operated 28 times).

\section{Statistics for the Substation B}

The total length of the network is $209 \mathrm{~km}$, out of which overhead lines cover $141 \mathrm{~km}$ with 82 secondary distribution substations $10 / 0.4 \mathrm{kV}$. The length of the cable network is $68 \mathrm{~km}$ with 108 secondary distribution substations $10 / 0.4 \mathrm{kV}$.

A total number of 61 interruptions lasting longer than 3 minutes were recorded in 2005 (over current protection has operated 29 times and short circuit protection has operated 12 times, in other cases it was not recorded which type of protection has operated). The total number of interruptions has increased to 81 in 2006 (over current protection has operated 59 times and short circuit protection has operated 11 times).

\section{EARTHING SYSTEMS CONDITION}

In order to assess the current condition of earthing systems in distribution substations $10 / 0.4 \mathrm{kV}$ at these two distribution areas, extended field measurements were conducted on the randomly chosen 55 / 46 distribution substations $10 / 0.4 \mathrm{kV}$ representing $29 \%$ and $24 \%$ of the total number of $10 / 0.4 \mathrm{kV}$ substations, respectively. The selected substations were chosen along 4/7 overhaed lines and 2/2 cable lines. Earthing resistance was measured with UI method, at the frequency of $128 \mathrm{~Hz}$ (Fig. 3. and Fig. 4.).

Croatia adopted the standard HD 637 S1 "Power installations exceeding $1 \mathrm{kV}$ " in 2002. The allowed level of touch voltage during continuous operation in short circuit conditions according to the standard is $75 \mathrm{~V}$, which implies that earthing resistance values should be lower than 2,5 Ohm for TN-type LV-systems. Alternatively the introduction of additional strcutures, such as buildings, fences or insulation asphalt layers is required to prevent too high touch voltages.

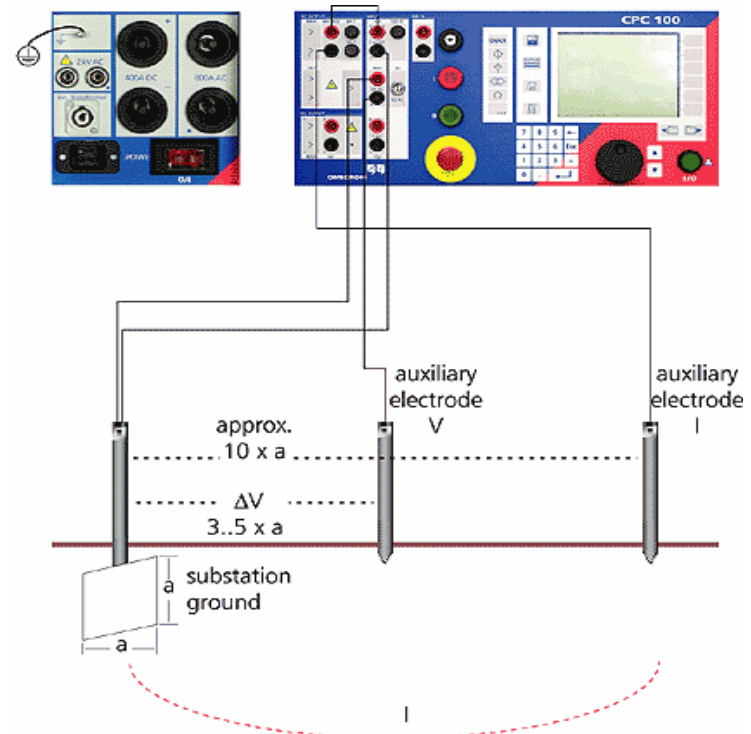

Fig. 3. Measuring procedure (UI method) and equipment

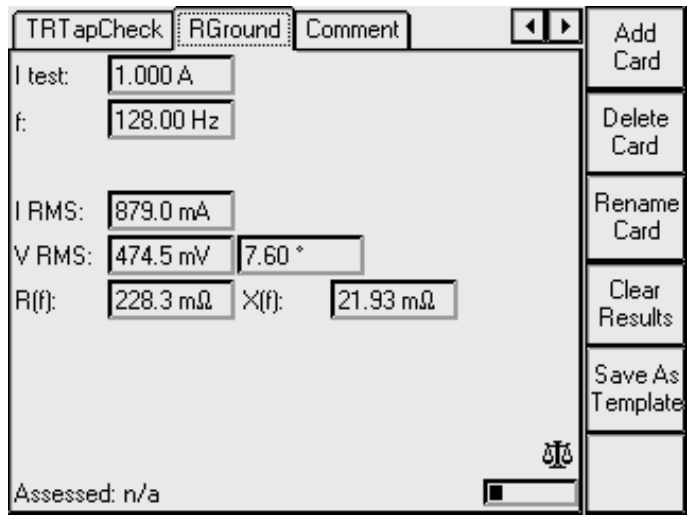

Fig. 4. Measuring window

An earthing resistance higher than 2,5 Ohm was measured in 12 out of 55 substations in area A, and in the 10 out of 46 substations in area B. All earthing systems in which earthing resistance exceeds the allowed values should be reconstructed before putting the Peterson coil in operation. In resonantly earthed MV networks, due to relatively small short circuit currents that flow in such conditions, uninterrupted operation can be continued for two hours. If the earthing systems are not properly earthed, touch voltages can be two fold higher than allowed ones and could therefore reach dangerous levels, threatening the safety of the network.

Earthing resistance control measurements are conducted every five years in all 10/0.4 kV distribution substations. All substations in the cable network had very low earthing resistance around $60 \mathrm{mOhm}$ and in all cases below $1 \mathrm{Ohm}$. According to obligatory safety measures, earthing systems that have higher than allowed resistance values have to be reconstructed before putting the Peterson coil in operation. 
If the earthing system is not properly constructed and considering that the fault condition in a resonantly earthed network can last for about two hours (due to small short circuit currents that flow in the network), there is a higher probability that people or animals (both mentioned distributions cover rural areas) enter into the potential field and be harmed by high touch or step voltages. Very high earthing resistance values of 10 and 19 Ohm were measured only in two substations.

\section{TESTING}

Before putting a Petersen coil in operation, it is necessary to record the resonance characteristics of the healthy network (Fig. 5.). The main parameters that determine these characteristics are:

$>\mathrm{U}_{\mathrm{res}}$ - maximum voltage $\mathrm{U}_{\mathrm{o}}$ in the resonance point;

$>\mathrm{I}_{\text {res }}$ - associated current of the Petersen coil for $\mathrm{U}_{\text {res; }}$;

$>\mathrm{I}_{\mathrm{W}}$ - wattmetric current across the fault location in the event of the low-resistance earth fault

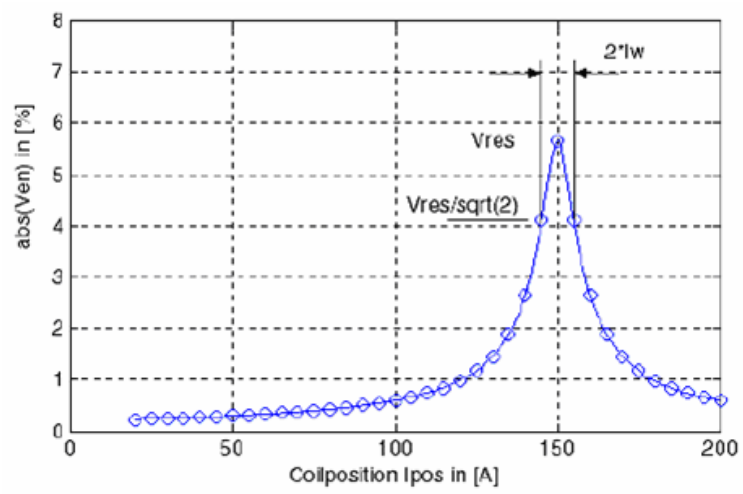

Fig. 5. Resonance characteristic

From these parameters attenuation of the system may be calculated as: $d=\frac{I_{w}}{I_{\text {res }}}$.

In addition to recording of resonance characteristics, it is necessary to test the proper functioning of the protection. Protection testing should be conducted for the cases of the single phase earth fault on the busbar in the substation and in the cases of the single phase earth fault in some distant point in the network. For both cases three distinctive tests should be conducted: for a direct single phase earth fault, an earth fault with resistance higher than $4 \mathrm{kOhms}$ and an intermitted earth fault. Protection testing during direct the single phase earth fault should be conducted for the cases of under- and overcompensation of $5 \%$ and $10 \%$.

The following values should be recorded: phase voltages (in the measuring field), neutral voltages (in the measuring field), and the Petersen coil neutral current and voltage. In the cases of the single phase earth fault on the busbar neutral currents of the transmission lines and transformer phase currents should be recorded (Fig. 6).

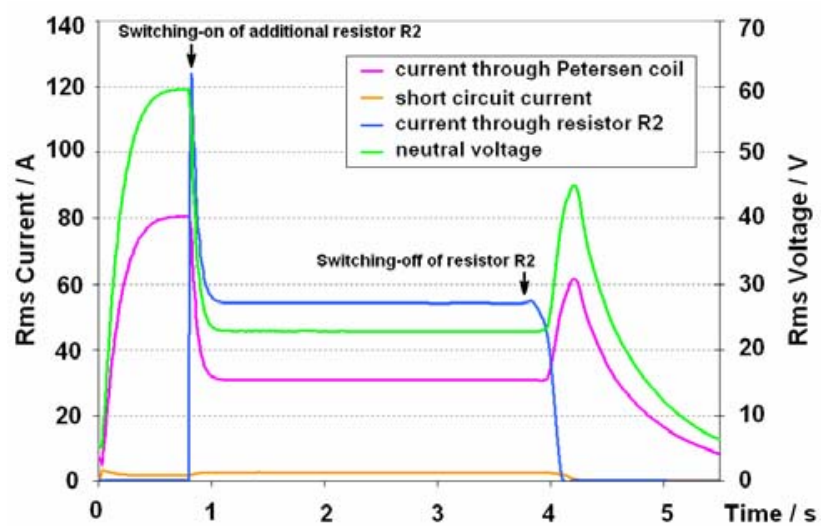

Fig. 6. Recorded transmission lines and transformer phase currents during single phase earth fault test (with additional resistor in parallel to Petersen coil)

Protection signals have to be recorded as well in order to clearly establish protection operation (trip or fail).

\section{PROTECTION}

The Wattmetric protection procedure is the predominantly used method for resonantly earthed medium voltage networks. However due to small residual currents, that flow in the compensated network during an earth fault, this type of protection can function properly only in case of earth faults with resistance up to $3-4 \mathrm{kOhm}$, even if a resistor is added in parallel to the Peterson coil, thus increasing the resistive (active) component (Fig. 6.).

The Wattmetric protection sensitivity depends on the adjusted operation level (threshold). Neutral voltage in the healthy network can reach values between $10-20 \%$ of the phase voltage. This voltage is caused due to incomplete transposition of the lines. In order to avoid false signals from short circuit protection, signalization should be allowed only if neutral point voltage reaches value between 20-30 \% of the phase voltage. Neutral voltage under the threshold value could be caused due to an earth fault with high resistance.

Additional protection methods could be used as well, such as harmonic procedure, harmonic procedure with ripple control systems, pulse locating and transient earth fault procedure [3].

\section{Two-phase-to-Earth Faults}

It is well known that during a solid phase-to-ground fault $\left(R_{F}=0\right)$, phase voltages of the two remaining un-faulted phases increase for the factor of $\sqrt{3}$ and are equal to the phase-to-phase voltages (Fig. 7.). This stresses the insulation of these phases and could result in a two- or three-phase-to-ground fault. The neutral-to-ground voltage equals the negative of the source phase-to-neutral voltage corresponding to the faulted phase. 


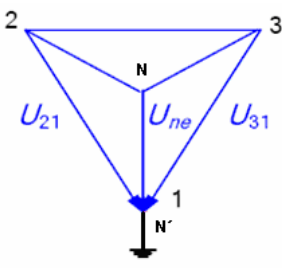

Fig. 7. Phase voltages during solid phase-to-ground fault

The probability for development of double earth faults at the weak points in the network is increased due to increased overvoltages [4]. Double earth fault can develop either on the second phase of the same line or in some other place in the network (on the other feeder line). In the case of the double earth fault of the same line, protection will switchoff only the faulted line (Fig. 8.). In case of double earth fault on two different lines overcurrent protection will switch-off both lines in less than $100 \mathrm{~ms}$.

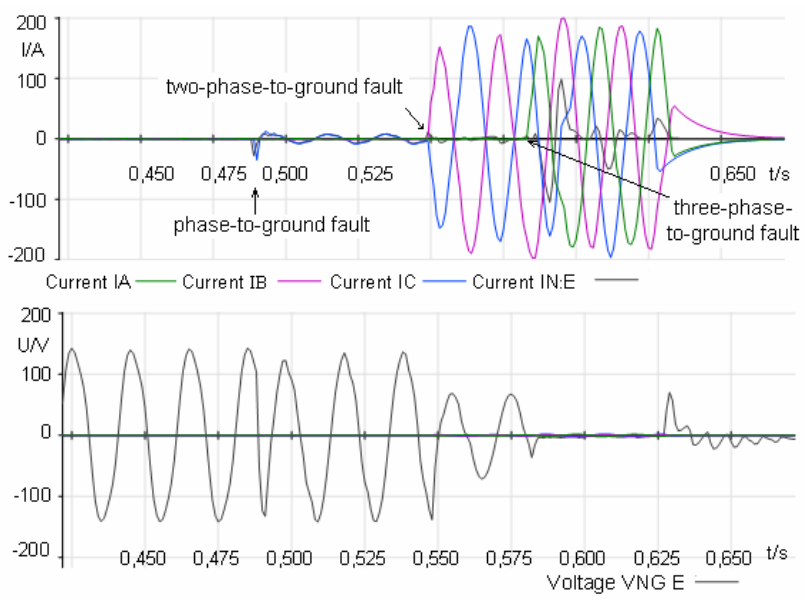

Fig. 8. An example of recorded development of double and three phase-to-ground earth fault

The equipment insulation level has to be considered in order to estimate if the installed equipment could sustain these overvoltages. All equipment in $110 \mathrm{kV}$ switch gear has an Si 123 insulation level and in $10 \mathrm{kV}$ switch gear has an Si
24 insulation level, which will insure safe operation even during double earth faults.

\section{CONCLUSION}

Earthing system evaluation of the secondary distribution substations $10 / 0.4 \mathrm{kV}$ should insure that safety regulations are followed. Before putting the Peterson coil in operation all systems that have higher than allowed resistance values, should be reconstructed, in order to ensure safe operation.

Conducted measurements have shown that about $10 \%$ of earthing systems in MV/LV distribution substations in a given distribution area will need some kind of intervention (improvement). This should therefore be taken into budgetary considerations.

Protection testing should also be performed before putting the Peterson coil in operation. Wattmetric protection can function properly only in case of an earth fault resistance up to 3-4 $\mathrm{kOhm}$.

\section{REFERENCES}

[1] A. Cerretti, G. Di Lembo, G. Valtorta, 2005, "Improvement in the Continuity of Supply Due to a Large Introduction of Petersen Coils in HV/MV Substations ", Proceedings CIRED Turin.

[2] DIN VDE 0228, Part 2, 1987, Actions Required for the Interference of Telecommunications Systems by Electrical Power Installations.

[3] Druml, 2007, Earth Fault Locating Relay EOR - D, Operating Manual, A.Eberle GmbH \& Co. KG.

[4] I. Zamora, A.J. Mazón,K.J. Sagastabeitia,O. Picó, J.R. Saenz, 2002, "Verifying Resonant Grounding in Distribution Systems", IEEE Computer Applications in Power, vol. 15, 45-50. 